\title{
INTERMEDIATE-AGE STARS AS ORIGIN OF THE LOW-VELOCITY DISPERSION NUCLEAR RING IN Mrk 1066
}

\author{
Rogemar A. Riffel ${ }^{1,2}$, Thaisa Storchi-Bergmann ${ }^{2}$, Rogério Riffel ${ }^{2}$, And Miriani G. Pastoriza ${ }^{2}$ \\ ${ }^{1}$ Departamento de Física, Centro de Ciências Naturais e Exatas, Universidade Federal de Santa Maria, 97105-900 Santa Maria, RS, Brazil; rogemar@ @mail.ufsm.br \\ ${ }^{2}$ Universidade Federal do Rio Grande do Sul, IF, CP 15051, 91501-970 Porto Alegre, RS, Brazil \\ Received 2010 January 14; accepted 2010 February 17; published 2010 March 23
}

\begin{abstract}
We report the first two-dimensional stellar population synthesis in the near-infrared of the nuclear region of an active galaxy, namely, Mrk 1066. We have used integral field spectroscopy with adaptative optics at the Gemini North Telescope to map the age distribution of the stellar population in the inner $300 \mathrm{pc}$ at a spatial resolution of $35 \mathrm{pc}$. An old stellar population component (age $\gtrsim 5 \mathrm{Gyr}$ ) is dominant within the inner $\approx 160 \mathrm{pc}$, which we attribute to the galaxy bulge. Beyond this region, up to the borders of the observation field $(\sim 300 \mathrm{pc})$, intermediate-age components $(0.3-0.7 \mathrm{Gyr})$ dominate. We find a spatial correlation between this intermediate-age component and a partial ring of low stellar velocity dispersions $\left(\sigma_{*}\right)$. Low- $\sigma_{*}$ nuclear rings have been observed in other active galaxies and our result for Mrk 1066 suggests that they are formed by intermediate-age stars. This age is consistent with an origin for the low- $\sigma_{*}$ rings in a past event which triggered an inflow of gas and formed stars which still keep the colder kinematics (as compared to that of the bulge) of the gas from which they have formed. At the nucleus proper we detect, in addition, two unresolved components: a compact infrared source, consistent with an origin in hot dust with mass $\approx 1.9 \times 10^{-2} M_{\odot}$, and a blue featureless power-law continuum, which contributes with only $\approx 15 \%$ of the flux at $2.12 \mu \mathrm{m}$.
\end{abstract}

Key words: galaxies: individual (Mrk 1066) - galaxies: nuclei - galaxies: Seyfert - galaxies: stellar content infrared: galaxies

Online-only material: color figures

\section{INTRODUCTION}

Optical spectroscopy on scales of hundreds of parsecs around the nucleus of Seyfert galaxies has shown that in $\approx 40 \%$ of them the active galactic nucleus (AGN) and young stars coexist (e.g., Storchi-Bergmann et al. 2000, 2001; González Delgado et al. 2001; Cid Fernandes et al. 2004; Asari et al. 2007; Dors et al. 2008), providing support to the so-called AGN-starburst connection (e.g., Norman \& Scoville 1988; Terlevich et al. 1990; Heckman et al. 1997; Heckman 2004; Riffel et al. 2009b). In particular, these authors have pointed out that the main difference between the stellar population (hereafter SP) of active and non-active galaxies is an excess of mainly intermediate-age stars in the former.

A similar result has been found in recent SP studies in the near-infrared (hereafter near-IR), using the technique of spectral synthesis (Riffel et al. 2009a, 2007). Using integrated spectra of the central few hundreds of parsecs in a sample of 24 Seyfert galaxies, these authors have shown that the continuum is dominated by the contribution of intermediate-age stellar population components (SPCs). In addition, they found that the near-IR nuclear spectra of about $50 \%$ of the Seyfert 1 and $~ 20 \%$ of the Seyfert 2 galaxies show emission from hot dust (Riffel et al. 2009a, 2009b, 2009c; Rodríguez-Ardila \& Mazzalay 2006; Rodríguez-Ardila et al. 2005).

Using a somewhat distinct technique, Davies et al. (2007) obtained near-IR integral field spectroscopy to investigate the circumnuclear star formation in nine nearby Seyfert galaxies at spatial resolutions of tens of parsecs. They have modeled the $\mathrm{Br} \gamma$ equivalent width, supernova rate, and mass-to-light ratio to quantify the star formation history in the center of these galaxies using their code STARS. They found that the ages of the stars which contribute most to the near-IR continuum lie in the range
10-30 Myr, but point out that these ages should be considered only as "characteristic," as they have not performed a proper spectral synthesis, arguing that there may be simultaneously two or more SPs that are not coeval (Davies et al. 2007, 2006).

In this paper we present, for the first time, two-dimensional (hereafter 2D) SP synthesis in the near-IR for the inner hundreds of parsecs of an active galaxy-namely Mrk 1066-using integral field spectroscopy with adaptive optics, which allowed us to derive the contribution of distinct SPCs to the nearIR spectra and map their spatial distributions. This paper is organized as follows. In Section 2 we describe the observations, data reduction procedures, and the spectral synthesis method; in Section 3 we present our results, which are discussed in Section 4. The conclusions are presented in Section 5.

\section{THE DATA AND SYNTHESIS CODE}

\subsection{Mrk 1066}

Mrk 1066 is an SB0 galaxy harboring a Seyfert 2 nucleus and located at a distance of $48.6 \mathrm{Mpc}$, for which $1^{\prime \prime}$ corresponds to $235 \mathrm{pc}$ at the galaxy. Previous studies of the central region of Mrk 1066 have shown that line emission from high-excitation gas is dominated by gas in a bi-conical outflow oriented along the position angle (P.A.) $=135 / 315^{\circ}$, which seems to be associated with the radio jet, while the low-excitation gas is more restricted to the plane of the galaxy (Bower et al. 1995; Nagar et al. 1999; Knop et al. 2001; Riffel et al. 2010; Riffel \& Storchi-Bergmann 2010). We selected this galaxy for the present study because it shows a signature of the presence of young/intermediateage stellar population in its stellar kinematics in the inner few hundred parsecs as illustrated in the stellar velocity dispersion $\left(\sigma_{*}\right)$ map shown in the top left panel of Figure 2. This map was obtained from the fit of the $\mathrm{CO}$ band heads around $2.3 \mu \mathrm{m}$ by 
a combination of stellar spectral templates from Winge et al. (2009) using the penalized Pixel-Fitting (pPXF) method of Cappellari \& Emsellem (2004). It shows a partial ring of low $\sigma_{*}$, a signature of the presence of stars which still have the kinematics of the cold gas from which they have formed. ${ }^{3}$ Our first goal with the spectral synthesis is to derive the age of this population. Additionally, high spatial resolution spectroscopy has shown that the near-IR nuclear continuum is consistent with a large contribution from hot dust emission (Riffel et al. 2010), not detected in lower resolution observations (Riffel et al. 2009a). Our second goal is to quantify this contribution with spectral synthesis.

\subsection{Observations and Data Reduction}

Mrk 1066 was observed with the Gemini Near-Infrared Integral-Field Spectrograph (NIFS; McGregor et al. 2003) operating with Gemini North Adaptive Optics system ALTAIR in 2008 September under the program GN-2008B-Q-30. Two set of observations were obtained, one at the $J$ band, covering a spectral region from $1.15 \mu \mathrm{m}$ to $1.36 \mu \mathrm{m}$ with a twopixel spectral resolving power of 6040, and another at the $K_{1}$ band at a resolving power of 5290 and covering the spectral range $2.1-2.5 \mu \mathrm{m}$.

The data reduction, described in Riffel et al. (2010), was performed using the GEMINI IRAF package and followed standard procedures. The resulting calibrated data cube contains 784 spectra covering an angular region of $2^{\prime \prime} .8 \times 22^{\prime \prime} .8$ at a sampling of $0^{\prime} .1 \times 00^{\prime \prime} 1$, which corresponds to $650 \times 650 \mathrm{pc}^{2}$ and $23.5 \times$ $23.5 \mathrm{pc}^{2}$, respectively. The angular resolution is $\approx 0$ '! 15 , corresponding to $35 \mathrm{pc}$ at the galaxy.

\subsection{Analysis}

In order to model the continuum and stellar absorption features we used the STARLIGHT spectral synthesis code (Cid Fernandes et al. 2004, 2005, 2009; Mateus et al. 2006; Asari et al. 2007). According to the authors STARLIGHT mixes computational techniques originally developed for semiempirical population synthesis with ingredients from evolutionary synthesis models.

In summary, the code fits an observed spectrum $O_{\lambda}$ with a combination, in different proportions, of $N_{*}$ single stellar populations (SSPs). Following Riffel et al. (2009a) we use the Maraston (2005) Evolutionary Population Synthesis (EPS) models as base set (described below). This choice is due to the fact that Maraston's models include the effects of stars in the thermally pulsing-asymptotic giant branch phase, which is crucial to model NIR SP (see Riffel et al. 2006, 2007, 2008a, 2009a). Extinction is modeled by STARLIGHT due to foreground dust and parameterized by the $V$-band extinction $A_{V}$ (Cid Fernandes et al. 2004, 2005). Note that all the components are reddened by the same amount. We use the CCM (Cardelli et al. 1989) extinction law.

Basically, the code solves the following equation for a model spectrum $M_{\lambda}$ (Cid Fernandes et al. 2005):

$$
M_{\lambda}=M_{\lambda 0}\left[\sum_{j=1}^{N_{*}} x_{j} b_{j, \lambda} r_{\lambda}\right] \otimes G\left(v_{*}, \sigma_{*}\right),
$$

\footnotetext{
3 A detailed discussion of the stellar kinematics is presented in Riffel \& Storchi-Bergmann (2010).
}

where $b_{j, \lambda} r_{\lambda}$ is the reddened spectrum of the $j$ th SSP normalized at $\lambda_{0} ; r_{\lambda}=10^{-0.4\left(A_{\lambda}-A_{\lambda 0}\right)}$ is the reddening term; $M_{\lambda 0}$ is the synthetic flux at the normalization wavelength; $\vec{x}$ is the population vector; $\otimes$ denotes the convolution operator; and $G\left(v_{*}, \sigma_{*}\right)$ is the Gaussian distribution used to model the lineof-sight stellar motions, it is centered at velocity $v_{*}$ with dispersion $\sigma_{*}$.

The final fit is carried out with a simulated annealing plus Metropolis scheme, which searches for the minimum of the equation (Cid Fernandes et al. 2005):

$$
\chi^{2}=\sum_{\lambda}\left[\left(O_{\lambda}-M_{\lambda}\right) w_{\lambda}\right]^{2},
$$

where emission lines and spurious features are masked out by fixing $w_{\lambda}=0$.

The spectral base was constructed with EPS models of Maraston (2005) as in Riffel et al. (2009a) and comprises SSP synthetic spectra covering 12 ages $(t=0.01,0.03,0.05,0.1,0.3$, $0.5,0.7,1,2,5,9$, and $13 \mathrm{Gyr})$ and 4 metallicities $(Z=0.02$, $0.5,1,2 Z_{\odot}$ ). We also included blackbody (BB) functions for temperatures in the range $700-1400 \mathrm{~K}$ in steps of $100 \mathrm{~K}$ (Riffel et al. 2009a) and a power law $\left(F_{v} \propto v^{-1.5}\right)$ in order to account for possible contributions from dust emission and from a featureless continuum (FC), respectively, in regions close to the nucleus (e.g., Cid Fernandes et al. 2004).

\section{RESULTS}

In Figure 1, we present the results of the synthesis for two spectra extracted within an aperture of $00^{\prime} 1 \times 00^{\prime \prime} 1$, one at the nucleus (top panel) and the other at $1^{\prime \prime}$ southeast of it (bottom panel). The observed (black) and synthetic (red) spectra were normalized to unit at $2.12 \mu \mathrm{m}$. This wavelength was chosen because the $K$-band spectra present a higher signal-to-noise ratio than those in the $J$-band, and the spectral region near $2.12 \mu \mathrm{m}$ is free of emission/absorption lines (Riffel et al. 2008a). In the inner $0.2(45 \mathrm{pc})$ radius the contribution from a FC and from dust emission is important, as illustrated in the top panel of Figure 1, where the combined contribution of all SPCs is shown in blue, the FC as a dotted line, and the composite BB function (sum over all temperatures) as a dashed line. We point out that the synthetic spectra (sum of all components) were reddened by the reddening amount detected in the observed spectra.

Following Cid Fernandes et al. (2004), we have binned the contribution of the SPCs $\left(x_{j}\right)$ to the flux at $2.2 \mu \mathrm{m}$ into four age $(t)$ ranges: young $\left(x_{y}: t \leqslant 100 \mathrm{Myr}\right)$, youngintermediate $\left(x_{y i}: 0.3 \mathrm{Gyr} \leqslant t \leqslant 0.7 \mathrm{Gyr}\right)$, intermediate-old $\left(x_{i o}: 1 \mathrm{Gyr} \leqslant t \leqslant 2 \mathrm{Gyr}\right)$, and old $\left(x_{0}: 5 \mathrm{Gyr} \leqslant t \leqslant 13 \mathrm{Gyr}\right)$. The percent flux contribution at $2.12 \mu \mathrm{m}$ from stars of each age range is shown in Figure 2: on average, the youngintermediate SPCs dominate the continuum emission from the central region of Mrk 1066, but there is a significant variation over the NIFS field. While in regions farther than $r \sim 0.7-160 \mathrm{pc}$ from the nucleus-the contribution of the young-intermediate age stars reaches values of up to $100 \%$, closer to the nucleus its contribution is negligible. Within this region the stellar population is dominated by the old component, whose contribution reaches $\approx 50 \%$, followed by the young component, whose contribution reaches $\approx 30 \%$ of the flux at $2.12 \mu \mathrm{m}$. Also within this region, but unresolved by our observations (thus within 0 .' 15 from the nucleus) we find a contribution of up to $50 \%$ from the combined BB components. Finally, we have found a small contribution from the AGN FC component, 

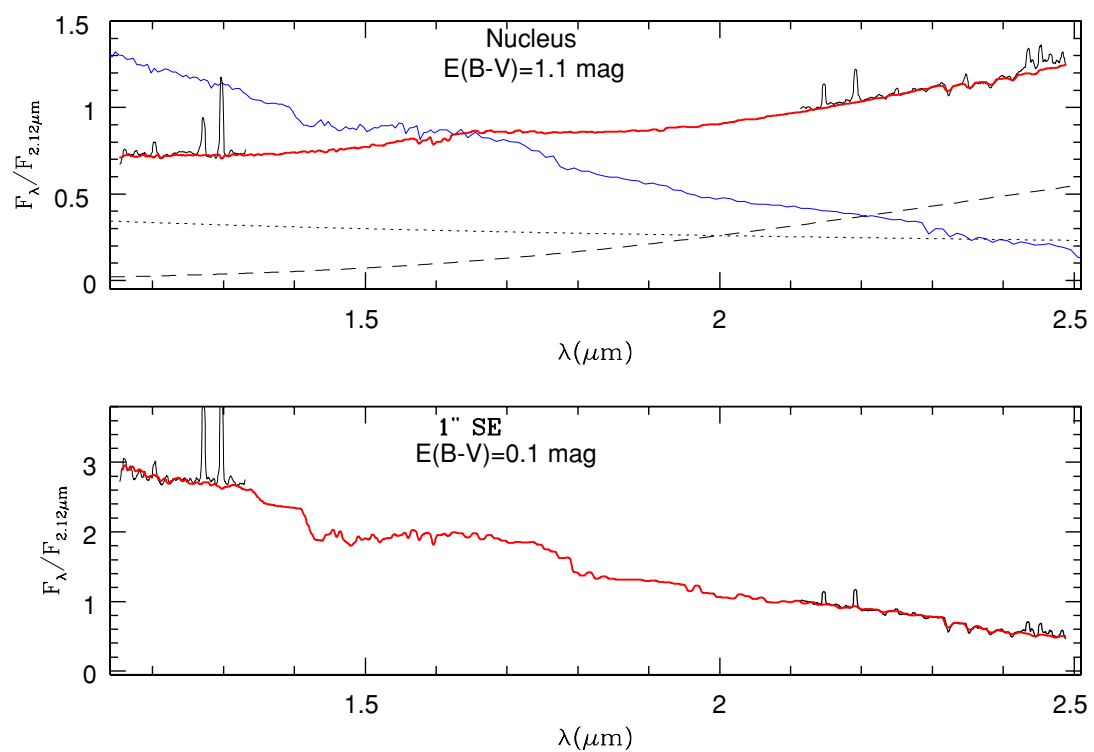

Figure 1. Sample fits of the SPCs to the nuclear spectrum (top) and to an extra-nuclear one at 1".0 southeast of the nucleus (bottom). The observed spectra are shown in black and the fits in red. In the top panel the dashed line shows the contribution of hot dust, the dotted line that of the featureless continuum, and the blue continuous line shows the contribution of the combined SPCs.

(A color version of this figure is available in the online journal.)
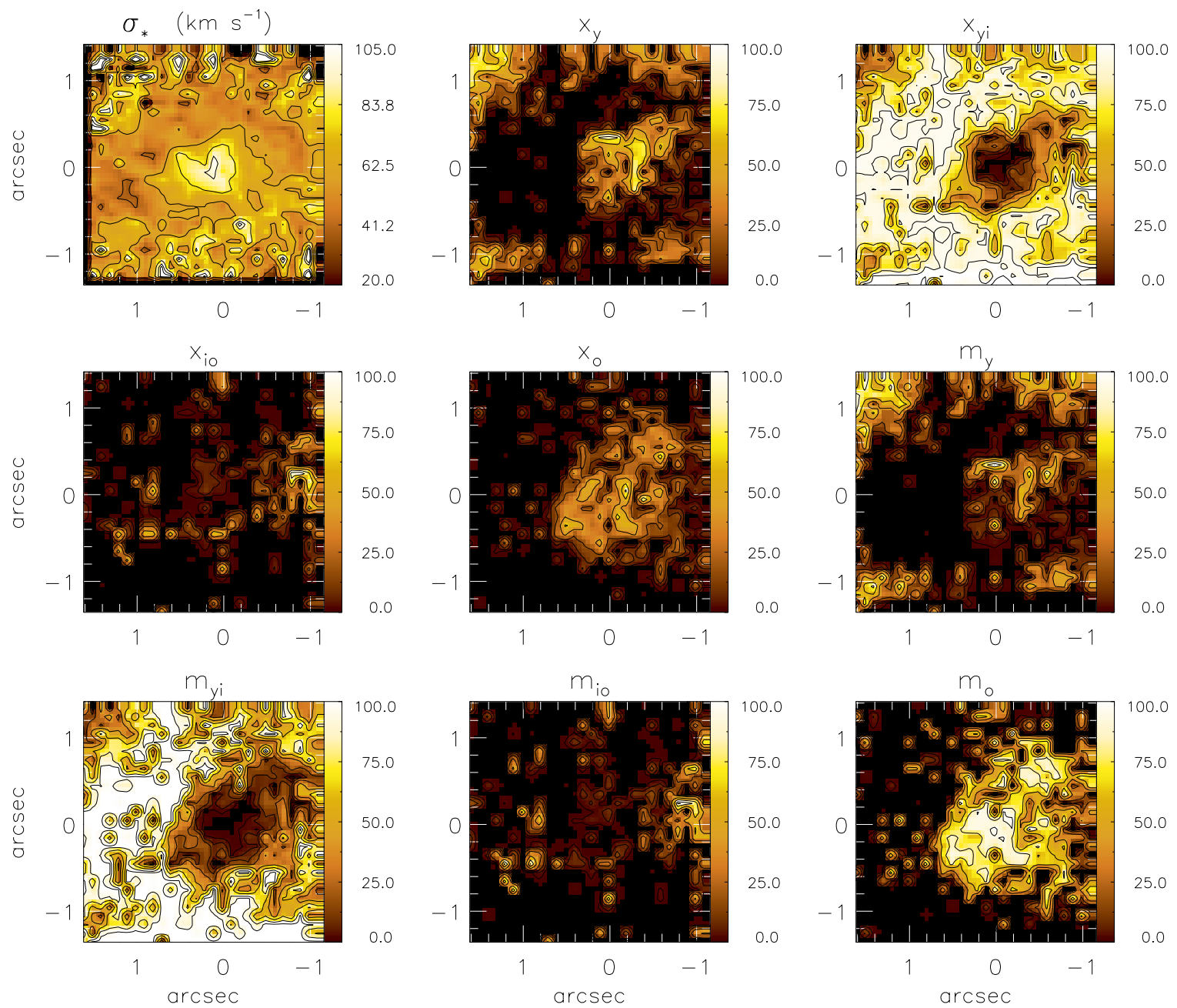

Figure 2. Stellar velocity dispersion map (top left panel) and spatial distributions of the percent contribution of each SPC to the flux at $\lambda=2.12 \mu \mathrm{m}\left(x_{j}\right)$ and to the mass $\left(m_{j}\right)$, where $j$ represents the age of the SPC: young $(y: \leqslant 100 \mathrm{Myr})$, young-intermediate (yi: 0.3-0.7 Gyr), intermediate-old (io: 1-2 Gyr), and old (o: 5-13 Gyr). (A color version of this figure is available in the online journal.) 

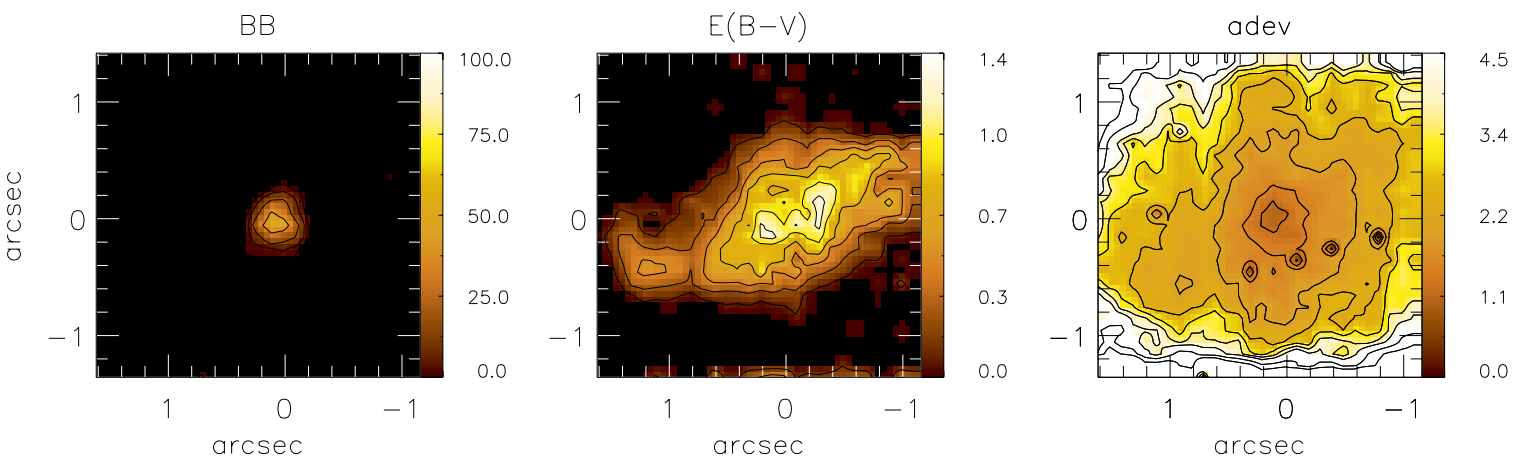

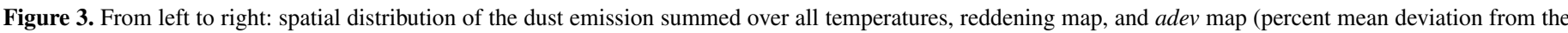
spectral fit).

(A color version of this figure is available in the online journal.)

which reaches at most $15 \%$ at the nucleus, also unresolved by our observations. The intermediate-old component contributes with less than $20 \%$ at most locations, with larger values more concentrated to the west-northwest of the nucleus.

Besides the SPC distributions, the synthesis outputs the average reddening of the stellar populations, as shown in the central panel of Figure 3. It reaches the highest values, of up to $E(B-V)=1.4$, along the P.A. $128^{\circ}$, which is the orientation of the line of nodes of the galaxy (Riffel \& Storchi-Bergmann 2010).

The robustness of the SP fit can be measured by the percent mean deviation (adev), $\left|O_{\lambda}-M_{\lambda}\right| / O_{\lambda}$, where $O_{\lambda}$ is the observed spectrum and $M_{\lambda}$ is the fitted model (Cid Fernandes et al. 2004, 2005). The resulting adev map is shown in the right panel of Figure 3 and presents values adev $\lesssim 2.5 \%$ at most locations, indicating that the model reproduces very well the observed spectra. Nevertheless, at regions close to the border of the NIFS field the adev reaches values of up to $5 \%$. At these locations we found an increase in the contribution of the young component $x_{y}$, which has thus larger uncertainties than in the rest of the NIFS field and should be further investigated with better data.

\section{DISCUSSION}

In general, our results are in good agreement with those of previous near-IR studies for single aperture nuclear spectra (Riffel et al. 2009a; Ramos Almeida et al. 2009). The main novelty of our work is the 2D mapping of the stellar population using near-IR spectra, which shows spatial variations in the contribution of the SPCs in the inner few hundred parsecs of Mrk 1066.

The significance of the above variations is further enhanced by the comparison with the $\sigma_{*}$ map presented in the top left panel of Figure 2. This map shows a partial ring of low- $\sigma_{*}$ values $\left(\approx 50 \mathrm{~km} \mathrm{~s}^{-1}\right)$ surrounding the nuclear region (which has $\sigma_{*} \approx 100 \mathrm{~km} \mathrm{~s}^{-1}$ ) at $\approx 1^{\prime \prime}$ from it. Such rings are commonly observed in the central region of active galaxies (Barbosa et al. 2006; Deo et al. 2006; Simões Lopes et al. 2007; Riffel et al. $2008 \mathrm{~b}, 2009 \mathrm{~b}$ ) and interpreted as being due to colder regions with more recent star formation than the underlying bulge. The comparison of the results from the SP synthesis of Mrk 1066 with the $\sigma_{*}$ map shows that the low- $\sigma_{*}$ value ring is associated with the young-intermediate age SPC, while the highest $\sigma_{*}$ values are associated with the old SPC, supporting the use of low stellar velocity dispersion as a tracer of younger stars in the bulge and confirming the interpretation of the above studies.

The flux-weighted SPC contributions depend on the normalization point and thus the comparison with results from other spectral regions should be done with caution. A physical parameter which does not depend on the normalization point and spectral range used in the synthesis is the mass of the stars. Thus, we constructed maps for the mass-weighted contribution of each SPC. The contributions for the young population $\left(m_{y}\right)$, young-intermediate population $\left(m_{y i}\right)$, intermediate-old population $\left(m_{i o}\right)$, and old population $\left(m_{o}\right)$ are shown in the rightmost panel of the second row, bottom left, bottom central, and bottom right panels of Figure 2, respectively. The mass-weighted contribution of the young population is very small over the whole field, while $m_{o}$ dominates within $\approx 160 \mathrm{pc}$ from the nucleus and $m_{y i}$ dominates in the circumnuclear ring.

The only previous 2D stellar population studies of active galaxies in the near-IR published to date are those from the group of R. I. Davies. In particular, Davies et al. (2007) investigated the circumnuclear star formation in nine Seyfert galaxies using near-IR integral field unit observations and also found circumnuclear disks of typical diameters of tens of parsecs with a "characteristic" age in the range 10-300 Myr based on measurements of the $\mathrm{Br} \gamma$ emission-line equivalent width, supernova rate, and mass-to-light ratio. Thus, the results we have found for Mrk 1066 are in reasonable agreement with those found by the Davies group. Nevertheless, the methodology adopted in the present work allowed us not only to obtain a "characteristic" age, but to also map, for the first time, the spatial distribution of stars of different ages in the central region of a Seyfert galaxy, on the basis of near-IR integral field spectroscopy.

As presented in Section 2.3, our spectral base includes four distinct metallicities: $Z=0.02,0.5,1,2 Z_{\odot}$. Following Cid Fernandes et al. (2004), we can estimate the mean flux and mass-weighted metallicity in the fit by

$$
\left\langle Z_{*}\right\rangle_{L}=\sum_{j=1}^{N_{*}} x_{j} Z_{j}
$$

and

$$
\left\langle Z_{*}\right\rangle_{M}=\sum_{j=1}^{N_{*}} m_{j} Z_{j},
$$

where $\left\langle Z_{*}\right\rangle_{L}$ is the flux-weighted mean metallicity, $\left\langle Z_{*}\right\rangle_{M}$ is the mass-weighted mean metallicity, and $N_{*}$ is the number of SSPs in the spectral base. In the case of Mrk 1066, we obtain a nearly solar metallicity- $\left\langle Z_{*}\right\rangle_{L}=0.021$ and $\left\langle Z_{*}\right\rangle_{M}=0.017-$ in good agreement with those found by Riffel et al. (2009a) from near-IR spectral synthesis using a single aperture nuclear spectrum. 
In further support of the results of the synthesis, we found that the average reddening map derived for the stellar population (the central panel of Figure 3) is in close agreement with the one derived for the narrow-line region using emissionline ratios (Riffel et al. 2010), presenting a similar S-shaped structure.

The synthesis also confirmed the presence of an unresolved $\mathrm{BB}$ component at the nucleus, which we had found in Riffel et al. (2010) from the fit of the nuclear spectrum by a BB function plus a power law. On the basis of its luminosity, we have followed the calculations as in Riffel et al. (2009a) to estimate a total dust mass of $M_{\mathrm{HD}} \approx 1.9 \times 10^{-2} M_{\odot}$, which is in good agreement with the value obtained in Riffel et al. (2010) and with those observed for other Seyfert galaxies (e.g., Riffel et al. 2009a, 2009b, 2009c; Rodríguez-Ardila \& Mazzalay 2006; RodríguezArdila et al. 2005).

As discussed in Section 3, we found that the FC component contributes with $\sim 15 \%$ of the $2.12 \mu \mathrm{m}$ nuclear continuum, which is in good agreement with the result found by Riffel et al. (2009a). This contribution is nevertheless uncertain, as it is hard to distinguish a reddened young starburst $(t \lesssim 5 \mathrm{Myr}$ ) from an AGN FC component (e.g., Storchi-Bergmann et al. 2000; Cid Fernandes et al. 2004; Riffel et al. 2009a). On the other hand, the contribution of the young population $x_{y}$ is dominated by stars with ages of 50-100 Myr, thus there does not seem to be a significant contribution from very young stellar populations, suggesting that the FC component originates from the emission of the AGN. This interpretation is supported by the detection of weak broad components for the $\mathrm{Pa} \beta$ and $\mathrm{Br} \gamma$ emission lines for the nuclear spectra of Mrk 1066 (Riffel et al. 2010; Veilleux et al. 1997). However, spectro-polarimetric studies show no evidence for the hidden Seyfert 1 nucleus in Mrk 1066 (e.g., Bian \& Gu 2007). Thus, the origin of the FC in Mrk 1066 must be further investigated.

We finally point out one caveat of the stellar population synthesis in the near-IR. In Riffel et al. (2010), on the basis of emission-line ratios, we have identified a large star-forming region at 0.5 southeast of the nucleus. In order to be active, this region should have an age $\lesssim 10$ Myr (Riffel et al. 2009b; Dors et al. 2008; Díaz et al. 2007; Kennicutt et al. 1989). Nevertheless, our synthesis did not find a young stellar component at this location, indicating that spectral synthesis in the near-IR (at least in the $J$ and $K$ bands) is not a good tracer of very recent star formation (age $\leqslant 10 \mathrm{Myr}$ ). This result is expected since the young (blue) population has its peak emission in ultraviolet/ optical wavelengths, while in the near-IR its contribution to the flux is very small, being hard to detect this component in this wavelength region.

In order to have a complete census of the SPCs, it is thus essential to study the stellar content of the central region of active galaxies using distinct methods and spectral regions.

\section{CONCLUSIONS}

The present work reports for the first time spectral synthesis in the near-IR with 2D coverage for the nuclear region of a Seyfert galaxy (Mrk 1066) within the inner $\approx 300$ pc at a spatial resolution of $\approx 35 \mathrm{pc}$. We have mapped the distribution of SPCs of different ages and of their average reddening. The main conclusions of this work are as follows.

1. The age of the dominant stellar population presents spatial variations: the flux and mass contributions within the inner $\approx 160$ pc are dominated by old stars $(t \geqslant 5$ Gyr), while intermediate-age stars $(0.3 \mathrm{Gyr} \leqslant t \leqslant 0.7 \mathrm{Gyr})$ dominate in the circumnuclear region.

2. There is a spatial correlation between the distribution of the intermediate-age component and low stellar velocity dispersion values which delineate a partial ring around the nucleus. Similar structures have been found around other active nuclei, and our result for Mrk 1066 suggests that these nuclear rings (and in some cases disks) are formed by intermediate-age stars.

3. There is an unresolved dusty structure at the nucleus with mass $M_{\mathrm{HD}} \approx 1.9 \times 10^{-2} M_{\odot}$, which may be the hottest part of the dusty torus postulated by the unified model of AGNs and a small contribution from a power-law continuum $(\approx 15 \%$ of the flux at $2.12 \mu \mathrm{m})$.

4. The near-IR synthesis seems not to be sensitive to very recent star formation (with $t \lesssim 5 \mathrm{Myr}$ ), reinforcing the importance of multi-wavelength stellar population studies of the central region of active galaxies.

This study is based on observations obtained at the Gemini Observatory, which is operated by the Association of Universities for Research in Astronomy, Inc., under a cooperative agreement with the NSF on behalf of the Gemini partnership: the National Science Foundation (United States), the Science and Technology Facilities Council (UK), the National Research Council (Canada), CONICYT (Chile), the Australian Research Council (Australia), Ministério da Ciência e Tecnologia(Brazil), and south-eastCYT (Argentina). This work has been partially supported by the Brazilian institutions CNPq and CAPES.

\section{REFERENCES}

Asari, N. V., Cid Fernandes, R., Stasińska, G., Torres-Papaqui, J. P., Mateus, A., Sodré, L., Schoenell, W., \& Gomes, J. M. 2007, MNRAS, 381, 263

Barbosa, F. K. B., Storchi-Bergmann, T., Cid Fernandes, R., Winge, C., \& Schmitt, H. 2006, MNRAS, 371, 170

Bian, W., \& Gu, C. 2007, ApJ, 657, 159

Bower, G., Wilson, A., Morse, J. A., Gelderman, R., Whitle, M., \& Mulchaey, J. 1995, ApJ, 454, 106

Cappellari, M., \& Emsellem, E. 2004, PASP, 116, 138

Cardelli, J. A., Clayton, G. C., \& Mathis, J. S. 1989, ApJ, 345, 245

Cid Fernandes, R., Gu, Q., Melnick, J., Terlevich, E., Terlevich, R., Kunth, D., Rodrigues Lacerda, R., \& Joguet, B. 2004, MNRAS, 355, 273

Cid Fernandes, R., Mateus, A., Sodré, L., Stasińska, G., \& Gomes, J. M. 2005, MNRAS, 358, 363

Cid Fernandes, R., et al. 2009, RevMexAA Conf. Ser., 35, 127

Davies, R. I., Sánchez, F. M., Genzel, R., Tacconi, L. J., Hicks, E. K. S., Friedrich, S., \& Sternberg, A. 2007, ApJ, 671, 1388

Davies, R. I., et al. 2006, ApJ, 646, 754

Deo, R. P., Crenshaw, D. M., \& Kraemer, S. B. 2006, AJ, 132, 321

Díaz, A. I., Terlevich, E., Castellanos, M., \& Hägele, G. F. 2007, MNRAS, 382, 251

Dors, O. L., Storchi-Bergmann, T., Riffel, R. A., \& Schmidt, A. 2008, A\&A, 482, 59

González Delgado, R., Heckman, T., \& Leitherer, C. 2001, ApJ, 546, 845

Heckman, T. M. 2004, in Co-evolution of Black Holes and Galaxies, from the Carnegie Observatories Centennial Symposia, ed. L. C. Ho (Carnegie Observatories Astrophysics Series; Cambridge: Cambridge Univ. Press), 358

Heckman, T. M., González Delgado, R. M., Leitherer, C., Meurer, G. R., Krolik, J., Wilson, A. S., Koratkar, A., \& Kinney, A. 1997, ApJ, 482, 114

Kennicutt, R. C., Jr., Keel, W. C., \& Blaha, C. A. 1989, AJ, 97, 1022

Knop, R. A., Armus, L., Matthews, K., Murphy, T. W., \& Soifer, B. T. 2001, AJ, 122,764

Maraston, C. 2005, MNRAS, 362, 799

Mateus, A., Sodré, L., Cid Fernandes, R., Stasińska, G., Schoenell, W., \& Gomes, J. M. 2006, MNRAS, 370, 721

McGregor, P. J., et al. 2003, Proc. SPIE, 4841, 1581

Nagar, N. M., Wilson, A. S., Mulchaey, J. S., \& Gallimore, J. F. 1999, ApJS, 120, 209

Norman, C., \& Scoville, N. 1988, ApJ, 332, 124 
Ramos Almeida, C., Pérez García, A. M., \& Acosta-Pulido, J. A. 2009, ApJ, 694, 1379

Riffel, R., Pastoriza, M. G., Rodriguez-Ardila, A., \& Bonatto, C. J. 2009a, MNRAS, 400, 273

Riffel, R., Pastoriza, M. G., Rodríguez-Ardila, A., \& Maraston, C. 2007, ApJ, 659,103

Riffel, R., Pastoriza, M. G., Rodriguez-Ardila, A., \& Maraston, C. 2008a, MNRAS, 388, 803

Riffel, R., Rodríguez-Ardila, A., \& Pastoriza, M. G. 2006, A\&A, 457, 61

Riffel, R. A., \& Storchi-Bergmann, T. 2010, MNRAS, submitted

Riffel, R. A., Storchi-Bergmann, T., Dors, O. L., \& Winge, C. 2009b, MNRAS, 393, 783

Riffel, R. A., Storchi-Bergmann, T., \& McGregor, P. J. 2009c, ApJ, 698, 1767

Riffel, R. A., Storchi-Bergmann, T., \& Nagar, N. 2010, MNRAS, in press (arXiv:1001.0130)
Riffel, R. A., Storchi-Bergmann, T., Winge, C., McGregor, P. J., Beck, T., \& Schmitt, H. 2008b, MNRAS, 385, 1129

Rodríguez-Ardila, A., Contini, M., \& Viegas, S. M. 2005, MNRAS, 357, 220

Rodríguez-Ardila, A., \& Mazzalay, X. 2006, MNRAS, 367, L57

Simões Lopes, R. D., Storchi-Bergmann, T., de Fátima Saraiva, M., \& Martini, P. 2007, ApJ, 655, 718

Storchi-Bergmann, T., González Delgado, R., Schmitt, H., Cid Fernandes, R., \& Heckman, T. 2001, ApJ, 559, 147

Storchi-Bergmann, T., Raimann, D., Bica, E. L. D., \& Fraquelli, H. A. 2000, ApJ, 544,747

Terlevich, E., Diaz, A. I., \& Terlevich, R. 1990, MNRAS, 242, 271

Veilleux, S., Goodrich, R. W., \& Hill, G. J. 1997, ApJ, 477, 631

Winge, C., Storchi-Bergmann, T., \& Riffel, R. A. 2009, ApJS, 185 186 